Int. J. Electrochem. Sci., 15 (2020) 10129 - 10140

Short Communication

\title{
Facile Synthesis of Hexagonal CdS Nanofilm on FTO Glass Substrate via Hydrothermal Method
}

\author{
Yang Tan ${ }^{\&, 1}$, Zheng Zhang ${ }^{\&, 1}$, Fuqiang Guo ${ }^{1, *}$, Renqing Guo ${ }^{2, *}$, Haineng Bai ${ }^{3, *}$, Baohua Zhang ${ }^{1}$, \\ Xin $\mathrm{Li}^{1}$, QianYang ${ }^{1}$, Xuebo Liu ${ }^{1}$ \\ ${ }^{1}$ Department of Physics, Changji University, Changji 831100, China \\ ${ }^{2}$ Department of Materials Engineering, Taizhou University, Taizhou 318000, China \\ ${ }^{3}$ National Laboratory of Solid State Microstructures, Nanjing University, Nanjing 210093, China \\ $\&$ These authors contributed equally to this work \\ *E-mail: cjxyedu@163.com, guorqing@126.com, bhn9052nju@163.com
}

doi: $10.20964 / 2020.10 .76$

Received: 5 July 2020 / Accepted: 19 August 2020 / Published: 31 August 2020

In this work, the compact CdS nanofilm has been deposited on FTO glass substrate with the conductive side facing down by the hydrothermal method. The microstructure and morphology of nanofilm were respectively analyzed by X-ray diffraction (XRD), scanning electron microscopy (SEM) and transmission electron microscopy (TEM). The results exhibited hexagonal wurtzite CdS nanofilm and continuously dense. Besides, the growth mechanism of CdS nanofilm was discussed and explored in detail: the small amount of $\mathrm{CdS}$ nuclei can crystallize during the initial reaction and form a CdS thin layer on the conductive side of FTO glass substrate; thereafter, with increasing temperature, many CdS nuclei can crystallize and grow on the CdS thin layer. Also, the photoelectric properties of nanofilm were studied by photoluminescence spectrum (PL), Raman spectrum, transient photocurrent response and electrochemical impedance spectroscopy (EIS).

Keywords: CdS nanofilm; hydrothermal method; CdS thin layer; FTO glass substrate; growth mechanism;

\section{$\underline{\text { FULL TEXT }}$}

(C) 2020 The Authors. Published by ESG (www.electrochemsci.org). This article is an open access article distributed under the terms and conditions of the Creative Commons Attribution license (http://creativecommons.org/licenses/by/4.0/). 